\title{
Association between prolonged breastfeeding and bone mineral density and osteoporosis in postmenopausal women: KNHANES 2010-2011
}

Sung-Woo Kim¹, Kwi-Hyun Bae ${ }^{1}$, Jae-Han Jeon ${ }^{1}$, Yeon-Kyung Choi ${ }^{1}$, Mi-Kyung Kim² ${ }^{2}$ Hye-Soon Kim ${ }^{2}$, Hyun-Ae Seo ${ }^{3}$, Eui Hyun Kim ${ }^{3}$, Keun-Gyu Park ${ }^{1}$ 1) Division of Endocrinology and Metabolism, Department of Internal Medicine, Kyungpook National University School of Medicine, Daegu, South Korea

2) Division of Endocrinology and Metabolism, Department of Internal Medicine, Keimyung University School of Medicine, Daegu, South Korea

3) Division of Endocrinology and Metabolism, Department of Internal Medicine, Daegu Fatima Hospital, Daegu, South Korea

Bone mineral density (BMD) is affected by various factors related to calcium homeostasis. Pregnancy and breastfeeding are associated with increase of maternal calcium loss and have a deleterious effect on osteoporosis. During pregnancy, approximately $30 \mathrm{~g}$ of calcium is demanded to develop the fetal skeleton until birth, and $300-400 \mathrm{mg}$ of maternal calcium is lost daily through breast milk during breastfeeding.

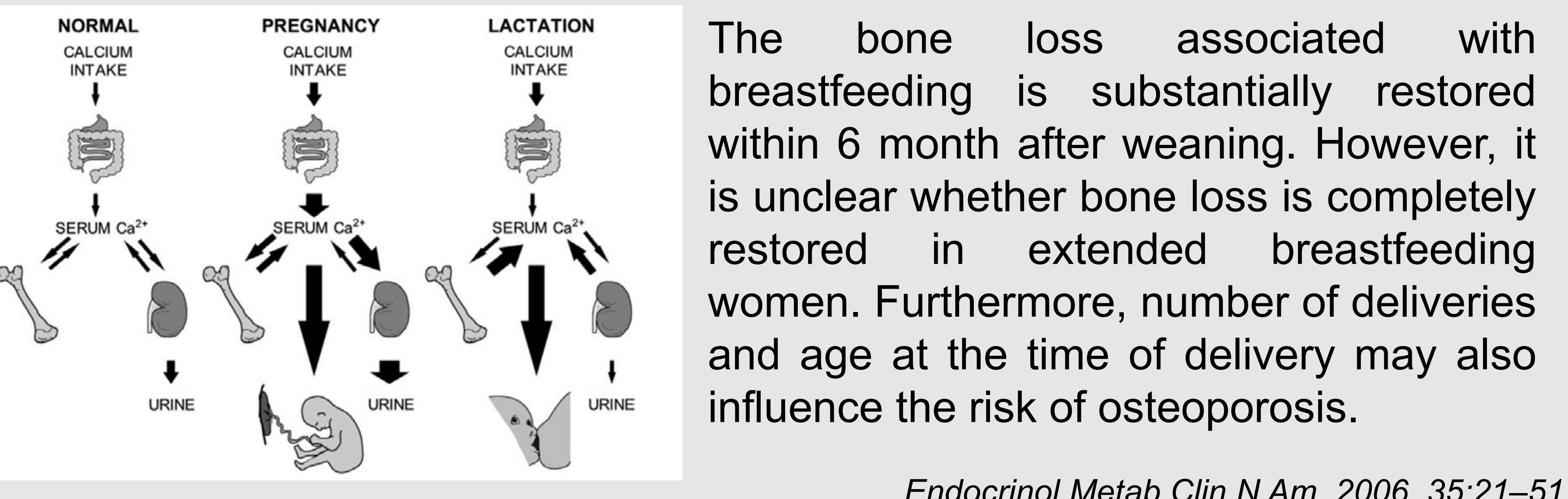

Endocrinol Metab Clin N Am. 2006. 35:21-51.

\section{Objectives}

The aim of this study was to examine the effects of breastfeeding on BMD and the prevalence of osteoporosis in postmenopausal women

\section{Method}

- The present study was based on KNHANES 2010 and 2011 data.

- Inclusion criteria : postmenopausal women over the age of 45

- Exclusion criteria : chronic renal disease, abnormal menopause, hormone replacement, osteoporosis treatment, incomplete data.

\section{Results}

1. Baseline characteristics of participants

\begin{tabular}{|c|c|c|c|c|}
\hline & $\begin{array}{l}\text { Total } \\
(n=1222)\end{array}$ & $\begin{array}{l}\text { Non-osteoporotic } \\
(n=797)\end{array}$ & $\begin{array}{l}\text { Osteoporotic } \\
(n=425)\end{array}$ & $P$ value \\
\hline Age, y & $62.8 \pm 8.8$ & $60.0 \pm 7.6$ & $68.1 \pm 8.5$ & $<0.001$ \\
\hline $\mathrm{BMI}, \mathrm{kg} / \mathrm{m}^{2}$ & $24.2 \pm 3.3$ & $24.7 \pm 3.4$ & $23.3 \pm 2.9$ & $<0.001$ \\
\hline Systolic BP, mmHg & $127.8 \pm 18.0$ & $128.4 \pm 17.9$ & $131.0 \pm 17.2$ & 0.012 \\
\hline Diastolic BP, mmHg & $76.2 \pm 10.2$ & $78.9 \pm 10.0$ & $76.4 \pm 10.0$ & $<0.001$ \\
\hline PTH, pg/mL & $69.2 \pm 29.2$ & $67.5 \pm 29.0$ & $72.4 \pm 29.3$ & 0.005 \\
\hline $25(\mathrm{OH}) \mathrm{D}_{3}, \mathrm{ng} / \mathrm{mL}$ & $17.5 \pm 6.9$ & $17.6 \pm 6.9$ & $17.4 \pm 6.9$ & 0.563 \\
\hline Lumbar spine BMD, $\mathrm{g} / \mathrm{cm}^{2}$ & $0.80 \pm 0.14$ & $0.87 \pm 0.10$ & $0.67 \pm 0.08$ & $<0.001$ \\
\hline Total femur BMD, $\mathrm{g} / \mathrm{cm}^{2}$ & $0.77 \pm 0.11$ & $0.82 \pm 0.09$ & $0.68 \pm 0.09$ & $<0.001$ \\
\hline Femoral neck BMD, g/cm² & $0.63 \pm 0.11$ & $0.67 \pm 0.09$ & $0.54 \pm 0.08$ & $<0.001$ \\
\hline DM, n (\%) & $144(11.8 \%)$ & $102(12.8 \%)$ & $42(9.9 \%)$ & 0.132 \\
\hline HTN, n (\%) & $492(40.3 \%)$ & $302(38.0 \%)$ & $190(44.7 \%)$ & 0.021 \\
\hline Duration of menopause, $y$ & $14.8 \pm 9.9$ & $11.5 \pm 8.1$ & $21.0 \pm 10.0$ & $<0.001$ \\
\hline Mean of breastfeeding duration, $m$ & $53.9 \pm 47.6$ & $44.1 \pm 42.2$ & $72.2 \pm 51.5$ & $<0.001$ \\
\hline \multicolumn{5}{|l|}{ Breastfeeding duration } \\
\hline 0-6 mon, n (\%) & $146(11.9 \%)$ & $119(14.9 \%)$ & $27(6.4 \%)$ & $<0.001$ \\
\hline 7-18 mon, n (\%) & $135(11.0 \%)$ & $113(14.2 \%)$ & $22(5.2 \%)$ & $<0.001$ \\
\hline $19-36$ mon, n (\%) & $337(27.6 \%)$ & $248(31.1 \%)$ & $89(20.9 \%)$ & $<0.001$ \\
\hline$\geq 37$ mon, n (\%) & $604(49.4 \%)$ & $317(39.8 \%)$ & $287(67.5 \%)$ & $<0.001$ \\
\hline Number of deliveries & $3.3 \pm 1.6$ & $3.0 \pm 1.4$ & $3.9 \pm 1.7$ & $<0.001$ \\
\hline Three or more deliveries, $\mathrm{n}(\%)$ & $778(63.7 \%)$ & $448(56.2 \%)$ & $330(77.6 \%)$ & $<0.001$ \\
\hline Age at first delivery, $y$ & $23.7 \pm 3.3$ & $24.0 \pm 3.3$ & $23.2 \pm 3.3$ & $<0.001$ \\
\hline First delivery age under $27, \mathrm{n}(\%)$ & $986(80.7 \%)$ & $633(79.4 \%)$ & $353(83.1 \%)$ & 0.168 \\
\hline Age at last delivery, y & $30.6 \pm 4.6$ & $29.8 \pm 4.3$ & $32.3 \pm 4.8$ & $<0.001$ \\
\hline Last delivery age over $35, \mathrm{n}(\%)$ & $234(19.1 \%)$ & $107(13.4 \%)$ & $127(29.9 \%)$ & $<0.001$ \\
\hline
\end{tabular}

All data are expressed as the mean \pm standard deviation or as number (\%).

All $P$ values were calculated using Student's t-test or the Chi-square test

Abbreviations: BMD, bone mineral density; BMI body mass index; BP, blood pressure; GFR, glomerular filtration rate; $P T H$, parathyroid hormone; DM, diabetes mellitus; HTN, hypertension *' $P<0.05$,
2. General characteristics of groups according to breastfeeding duration

\begin{tabular}{|c|c|c|c|c|c|}
\hline & \multicolumn{5}{|c|}{ Duration of breastfeeding } \\
\hline & $\begin{array}{l}\text { Group } 1 \\
(n=146)\end{array}$ & $\begin{array}{l}\text { Group } 2 \\
(n=135)\end{array}$ & $\begin{array}{l}\text { Group } 3 \\
(n=337)\end{array}$ & $\begin{array}{l}\text { Group } 4 \\
(\mathrm{n}=604)\end{array}$ & $P$ value \\
\hline $\begin{array}{l}\text { Duration of breastfeeding } \\
(\text { mean, } \mathrm{m})\end{array}$ & $1.3 \pm 2.1$ & $13.3 \pm 2.8$ & $29.1 \pm 5.8$ & $89.5 \pm 43.8$ & $<0.001$ \\
\hline Age, y & $56.8 \pm 6.7$ & $56.6 \pm 6.4$ & $58.9 \pm 6.8$ & $67.8 \pm 7.8$ & $<0.001$ \\
\hline $\mathrm{BMI}, \mathrm{kg} / \mathrm{m}^{2}$ & $23.6 \pm 3.2$ & $24.2 \pm 3.4$ & $23.8 \pm 3.0$ & $24.6 \pm 3.4$ & $<0.001$ \\
\hline Systolic BP, mmHg & $126.1 \pm 20.0$ & $125.4 \pm 18.7$ & $126.6 \pm 16.5$ & $132.4 \pm 17.0$ & $<0.001$ \\
\hline Diastolic BP, mmHg & $79.6 \pm 11.6$ & $78.7 \pm 10.2$ & $78.2 \pm 9.6$ & $77.4 \pm 9.9$ & 0.088 \\
\hline PTH, pg/mL & $68.4 \pm 31.7$ & $67.7 \pm 24.0$ & $65.1 \pm 24.9$ & $72.0 \pm 31.4$ & 0.007 \\
\hline $25(\mathrm{OH}) \mathrm{D}_{3}, \mathrm{ng} / \mathrm{mL}$ & $17.2 \pm 6.1$ & $16.8 \pm 6.4$ & $17.5 \pm 6.7$ & $17.8 \pm 7.2$ & 0.430 \\
\hline DM, n (\%) & $10(6.8 \%)$ & $14(10.4 \%)$ & $27(8.0 \%)$ & $93(15.4 \%)$ & 0.001 \\
\hline HTN, n (\%) & $40(27.4 \%)$ & $46(34.1 \%)$ & $106(31.5 \%)$ & $300(49.7 \%)$ & $<0.001$ \\
\hline Osteoporosis, n (\%) & $27(18.5 \%)$ & $22(16.3 \%)$ & $89(26.4 \%)$ & $287(47.5 \%)$ & $<0.001$ \\
\hline Lumbar spine fracture, $n(\%)$ & $0(0 \%)$ & $1(0.7 \%)$ & $2(0.6 \%)$ & $12(2.0 \%)$ & 0.026 \\
\hline Duration of menopause, $y$ & $8.7 \pm 7.7$ & $7.8 \pm 6.6$ & $10.5 \pm 7.3$ & $20.1 \pm 9.4$ & $<0.001$ \\
\hline Number of deliveries, $n$ & $1.9 \pm 1.3$ & $2.1 \pm 1.0$ & $2.6 \pm 0.9$ & $4.3 \pm 1.4$ & $<0.001$ \\
\hline Age at first delivery, $y$ & $26.2 \pm 3.4$ & $25.4 \pm 3.3$ & $24.6 \pm 2.9$ & $22.3 \pm 2.7$ & $<0.001$ \\
\hline Age at last delivery, y & $29.6 \pm 3.9$ & $29.0 \pm 4.5$ & $28.7 \pm 3.7$ & $32.3 \pm 4.6$ & $<0.001$ \\
\hline Duration from last delivery, $y$ & $27.0 \pm 7.6$ & $27.6 \pm 7.5$ & $30.2 \pm 7.1$ & $35.5 \pm 7.2$ & $<0.001$ \\
\hline
\end{tabular}

3. Association between breastfeeding duration and lumbar spine BMD and osteoporosis

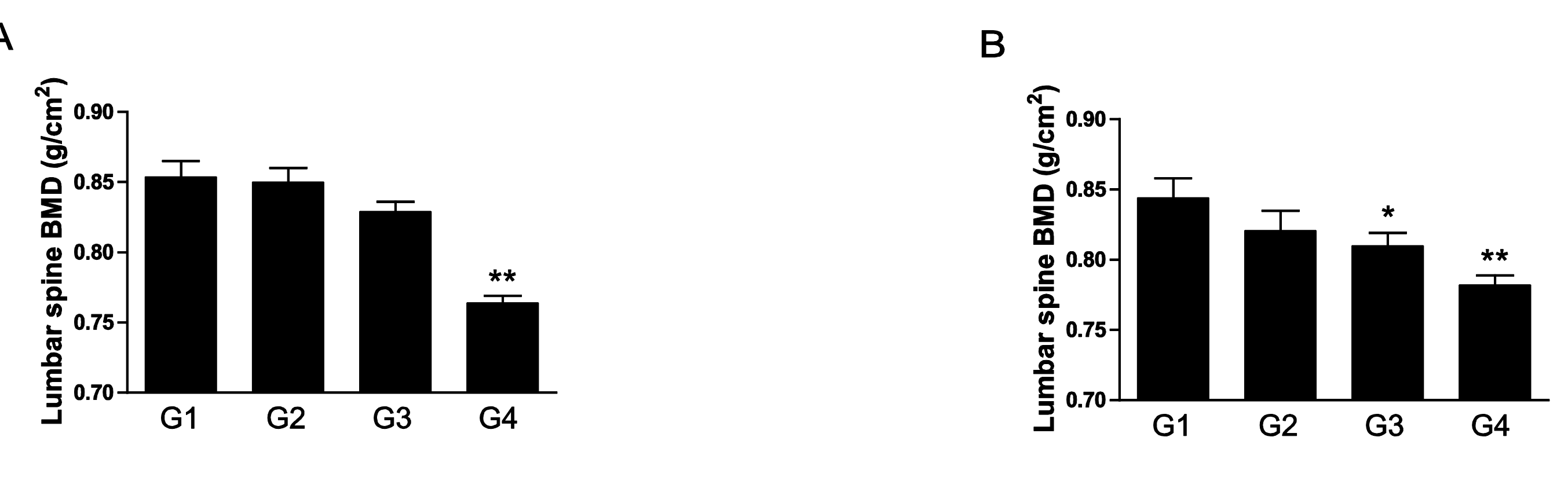

Association between breastfeeding duration and lumbar spine bone mineral density according to unadjusted $(A)$ and adjusted $(B)$ models.

\begin{tabular}{|c|c|c|c|c|c|}
\hline OR & $\begin{array}{l}0-6 \text { mon } \\
(n=146)\end{array}$ & $\begin{array}{l}7-18 \text { mon } \\
(n=135)\end{array}$ & $\begin{array}{l}19-36 \text { mon } \\
(\mathrm{n}=337)\end{array}$ & $\begin{array}{l}\geq 37 \text { mon } \\
(\mathrm{n}=604)\end{array}$ & $\mathrm{P}$ for trend \\
\hline & 1 (Ref) & $\begin{array}{l}0.858 \\
(0.462-1.594)\end{array}$ & $\begin{array}{l}1.582 \\
(0.976-2.564)\end{array}$ & $\begin{array}{l}3.990 * \star \\
(2.551-6.241)\end{array}$ & $<0.001$ \\
\hline Model 1 & 1 (Ref) & $\begin{array}{l}1.227 \\
(0.503-2.995)\end{array}$ & $\begin{array}{l}2.238 \text { * } \\
(1.105-4.532)\end{array}$ & $\begin{array}{l}2.586 * \star \\
(1.280-5.223)\end{array}$ & 0.003 \\
\hline Model 2 & 1 (Ref) & $\begin{array}{l}1.242 \\
(0.489-3.155)\end{array}$ & $\begin{array}{l}2.820 * * \\
(1.308-6.080)\end{array}$ & $\begin{array}{l}3.292 * * \\
(1.485-7.299)\end{array}$ & 0.001 \\
\hline
\end{tabular}

Association between breastfeeding duration and odds ratios for osteoporosis

4. The association between childbirth parameters and lumbar spine fracture

\begin{tabular}{|c|c|c|c|}
\hline & Unadjusted model & Model 1 & Model 2 \\
\hline $\begin{array}{l}\text { Duration of breastfeeding, } \\
\mathrm{m}\end{array}$ & $1.016(1.009-1.023)$ ** & $1.014(1.003-1.025)^{*}$ & $1.015(1.001-1.030)$ * \\
\hline Number of deliveries, $n$ & $1.497(1.172-1.913)$ ** & $1.310(0.890-1.928)$ & $0.999(0.537-1.861)$ \\
\hline Age at first delivery, $y$ & $0.964(0.820-1.133)$ & $1.074(0.904-1.276)$ & $1.145(0.915-1.434)$ \\
\hline Age at last delivery, $y$ & $1.177(1.079-1.283)^{* *}$ & $1.139(1.017-1.275)$ * & $1.078(0.921-1.262)$ \\
\hline \multicolumn{4}{|c|}{$\begin{array}{l}\text { Multivariable regression logistic model } 1 \text { : adjusted for age, BMI, age at menarche, duration of } \\
\text { menopause, systolic blood pressure, GFR, PTH, } 25(\mathrm{OH}) \mathrm{D}_{3} \text {, oral contraceptive use, HTN, DM, } \\
\text { physical activity, and alcohol and smoking status. } \\
\text { Multivariable regression logistic model } 2 \text { : adjusted for age, BMI, age at menarche, duration of } \\
\text { menopause, systolic blood pressure, GFR, PTH, } 25(\mathrm{OH}) \mathrm{D}_{3} \text {, oral contraceptive use, HTN, DM, } \\
\text { physical activity, alcohol and smoking status, number of deliveries, and age at first and last delivery. } \\
\text { All data are expressed as odds ratios (95\% confidence intervals). } \\
{ }^{*} P<0.05 .,{ }^{* \star} P<0.01 .\end{array}$} \\
\hline
\end{tabular}

\section{Conclusions}

-We found a negative correlation between duration of breastfeeding and BMD in the lumbar spine. Moreover, the prevalence of osteoporosis was significantly higher in those that breastfed for more than 18 months. Neither the number of deliveries nor age at the time of delivery was associated with BMD at any site.

Taken together, the results of the present study suggest that prolonged breastfeeding may be an important risk factor for osteoporosis. 